\title{
Enjeux d'hier dans les découpages territoriaux d'aujourd'hui : les Grimaldi de Monaco et le Carladez (Aveyron-Cantal)
}

Géraud Cullier de Labadie et Marie Redon

\section{(2) OpenEdition}

Journals

Édition électronique

URL : http://journals.openedition.org/echogeo/13980

DOI : 10.4000/echogeo. 13980

ISSN : 1963-1197

Éditeur

Pôle de recherche pour l'organisation et la diffusion de l'information géographique (CNRS UMR 8586)

Référence électronique

Géraud Cullier de Labadie et Marie Redon, «Enjeux d'hier dans les découpages territoriaux

d'aujourd'hui : les Grimaldi de Monaco et le Carladez (Aveyron-Cantal) », EchoGéo [En ligne], Sur le Vif, mis en ligne le 03 novembre 2014, consulté le 01 mai 2019. URL : http://journals.openedition.org/ echogeo/13980; DOI : 10.4000/echogeo.13980

Ce document a été généré automatiquement le 1 mai 2019.

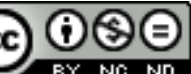

EchoGéo est mis à disposition selon les termes de la licence Creative Commons Attribution - Pas d'Utilisation Commerciale - Pas de Modification 4.0 International 


\title{
Enjeux d'hier dans les découpages territoriaux d'aujourd'hui : les Grimaldi de Monaco et le Carladez (Aveyron-Cantal)
}

\author{
Géraud Cullier de Labadie et Marie Redon
}

\section{Introduction}

1 Les 14 et 15 mai 2014, Son Altesse Sérénissime (S.A.S.) le prince Albert II de Monaco et son épouse se sont rendus en visite dans le Carladez, territoire correspondant au fief échu en 1643 à la famille Grimaldi. À présent scindé entre les départements du Cantal et de l'Aveyron, et donc les régions Auvergne et Midi-Pyrénées, la persistance de l'entité territoriale nommée Carladez ${ }^{1}$ fait question dans un contexte de remise en cause des découpages territoriaux de la République. Le 3 juin 2014, le gouvernement a en effet proposé une réforme de la carte des régions, première étape d'une rationalisation de ce millefeuille politico-administratif décrié car considéré comme pesant. Cette visite princière peut paraître fort anecdotique et relever davantage de la presse people que d'une revue de géographie. Pourtant, l'événement a paru offrir à l'historien comme à la géographe l'opportunité de sonder les enjeux tissés, dans le temps et l'espace, autour de ce questionnement : qu'est-ce qui donne sa légitimité à une entité territoriale?

2 Précisons tout d'abord que la visite de mai 2014 s'inscrit dans une série de visites aux anciens fiefs des Grimaldi dans le royaume de France : en Normandie en 2011 à Saint Lô, en Bretagne en 2012, dans le Valentinois en 2013 (voir illustration 1) 2. 
Illustration 1 - Localisation des fiefs des Grimalfi de Monaco provenant du traité de Péronne (1641) dans l'actuel territoire français

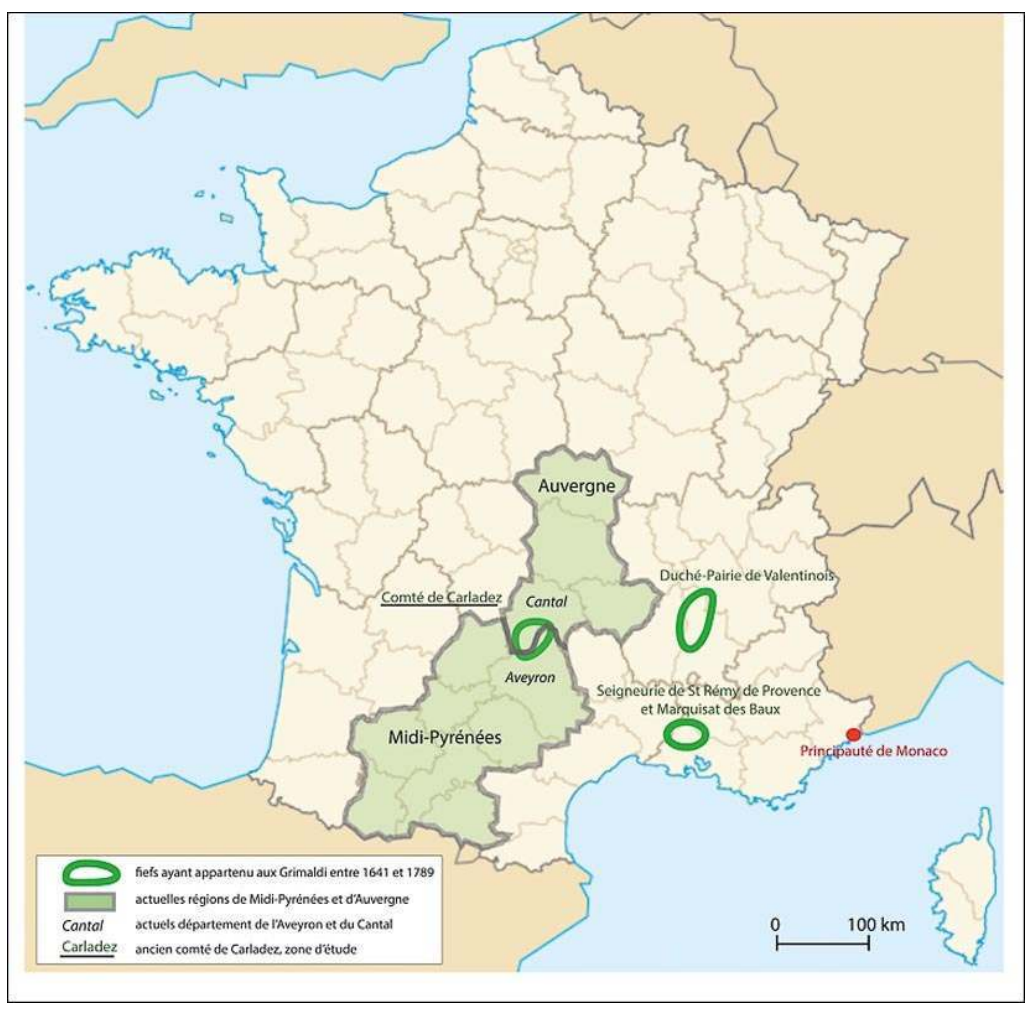

Pour le Carladez, le président du Conseil général du Cantal a lancé une invitation au Prince, ainsi que chaque commune concernée. Devant cette " convergence d'invitations », S.A.S. a décidé de venir et « il a ensuite fallu «mettre en musique » le tout et trouver un parcours cohérent $»^{3}$ (voir illustration 2). 


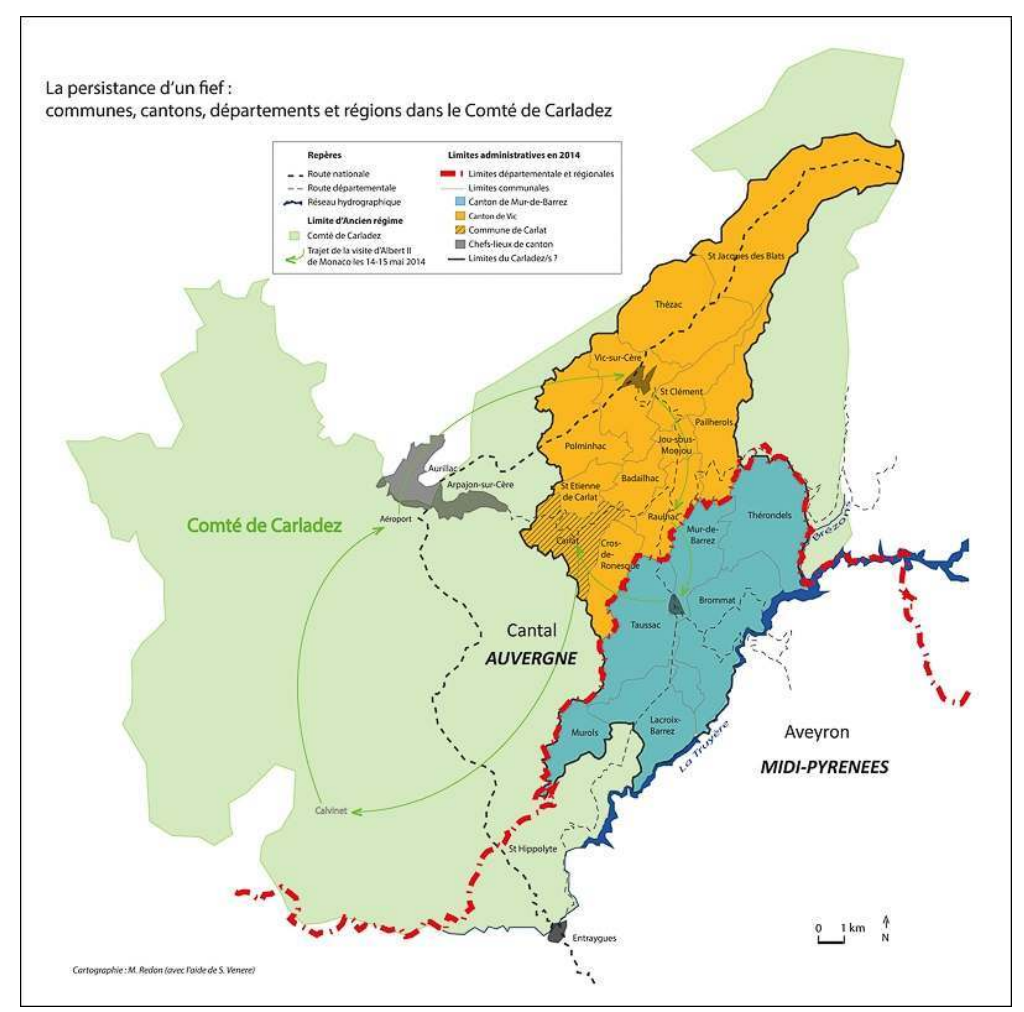

4 L'année 2014 correspondait, en outre, au centenaire de la restitution du rocher de Carlat au Prince ${ }^{4}$. Le propos de cet article est de comprendre en quoi l'ancien fief fait toujours sens, comme entité territoriale, malgré les découpages postérieurs. «Les découpeurs d'espace aiment «la géographie» [parce que] c'est un inépuisable fournisseur de légitimité », comme si « le support était légitime par son éternité, et par ce qui lui reste d'enchantement, les accidents du terrain et les cours d'eau ont encore leurs dieux enfouis au subconscient des amateurs" (Brunet, 1997, p. 252). L'éternité, certainement pas, l'ancrage historique peut-être d'où la question d'une éventuelle réactivation politique du Carladez. Dans un premier temps, il s'agira d'analyser comment une histoire commune a donné une forme de légitimité historique et d'ancrage territorial à cette entité, visible dans l'émergence d'une destination touristique. Cela nous mènera à aborder le Carladez comme exemple de tensions entre logiques fonctionnelles et découpages territoriaux.

5 Au-delà de l'observation sur place de la visite de mai 2014, l'éclairage donné ici repose sur une série d'entretiens semi-directifs menés avec des élus, des acteurs du tourisme local et des habitants des parties aveyronnaise et cantalienne du Carladez en mai et septembre 2014.

\title{
Le Carladez, une entité historique, une destination touristique
}

\author{
« Nos deux rochers, géographiquement séparés \\ mais historiquement liés...»
}


Albert de Monaco, discours effectué à Carlat le

15 mai 2014.

6 Nous ciblerons le propos sur la partie orientale de l'ancien fief qui correspond à l'actuel Carladez "vécu ", comme en témoignent les noms donnés aux intercommunalités et le trajet de la visite princière en mai 2014. Le Carladez est un pays dans le sens d'une «survivance d'unité spatiale aux contours mal définis, sans réelle valeur administrative sous l'Ancien régime " (Ardillier-Carras, 1999) auquel se rattachent 18 communes situées à cheval sur deux cantons, et deux départements : l'Aveyron et le Cantal. Le Carladez actuel comprend à la fois les 12 communes du canton de Vic-sur-Cère du côté Cantal et les 6 communes du canton de Mur-de-Barrez dans l'Aveyron (voir illustration 2). Statutairement, Monaco est une Principauté, État indépendant membre de l'ONU depuis 1993 et dirigée par S.A.S. le Prince Albert II depuis 2005. Indéniablement, ces deux territoires ont peu en commun. Pourtant, comme le déclarait le Prince le 14 mai dernier, "les liens du passé méritent d'être entretenus ", au point de se rendre sur place et de passer deux journées entre Aveyron et Cantal, dans l'ancien fief de la famille Grimaldi : mais qu'allait-il donc faire sur ces terres?

Illustration 3 - La Tour de Monaco sur la place principale de Mur-de-Barrez, en attendant Albert II

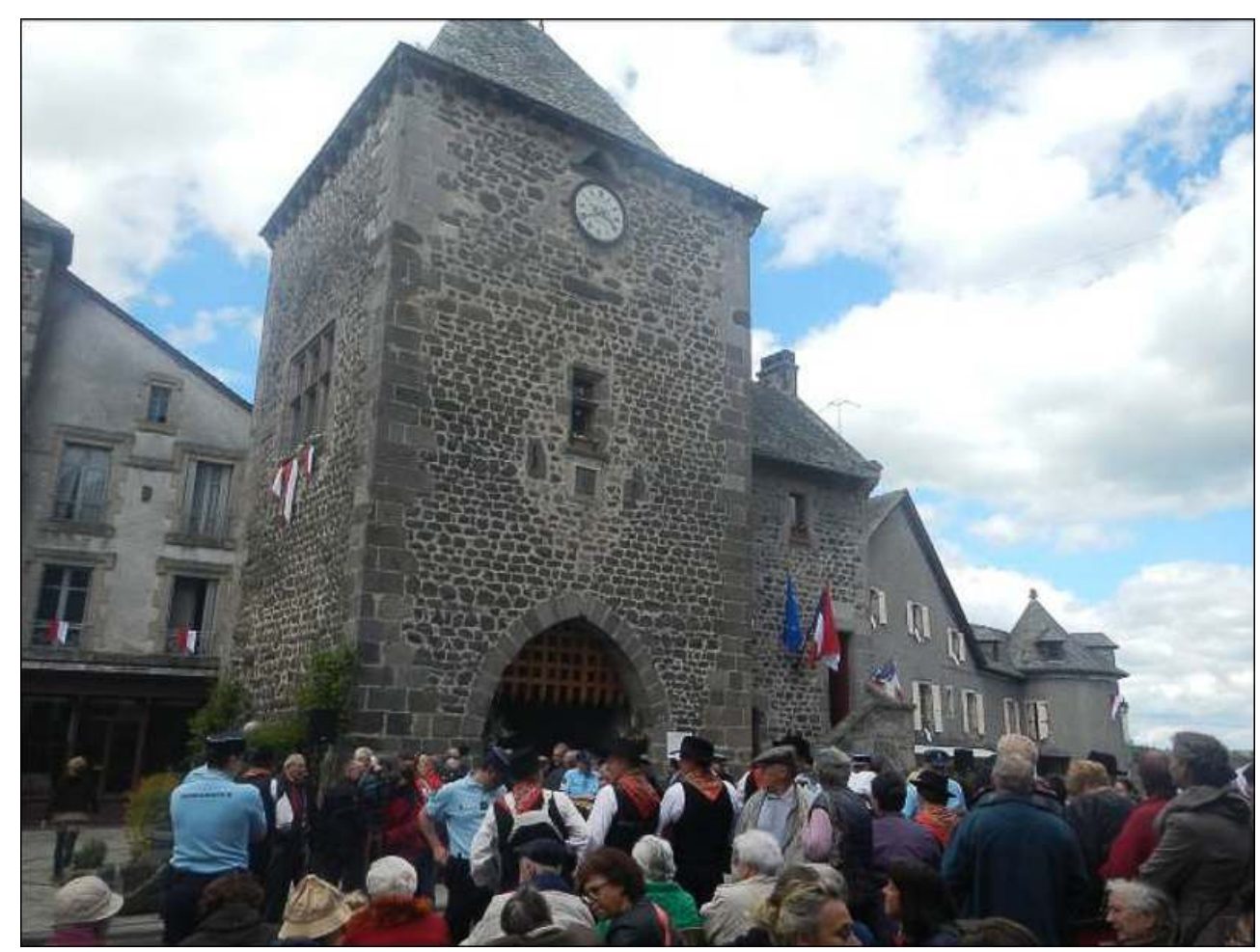

Auteur: M. Redon, 14 mai 2014.

\section{Le Carladez, ancien fief des Grimaldi de Monaco dans le royaume de France (1643-1789)}

7 Dès 1630-1631, le Prince Honoré II de Grimaldi avait entamé des négociations secrètes avec la France pour renverser l'alliance avec l'Espagne qu'il jugeait trop contraignante ${ }^{5}$. Ces pourparlers ${ }^{6}$ aboutirent au traité secret, conclu à Péronne le 14 septembre 1641, par 
lequel Honoré II devenait le vassal de Louis XIII et se plaçait sous sa protection militaire (voir illustration 4). Le traité ne deviendrait effectif que lorsque le Prince de Monaco aurait chassé les Espagnols de Monaco. Ce fut accompli le 17 novembre 1641. Dès lors, la confiscation de ses biens en pays espagnol devant être compensée, le roi de France lui octroya les fiefs suivants : le duché de Valentinois, le marquisat des Baux de Provence, la seigneurie de Saint Rémy-de-Provence, la vicomté de Carlat (voir illustration 1). Le traité de Péronne stipulait donc la donation de domaines dans le royaume de France d'un revenu égal à celui des possessions perdues en territoire espagnol. Les fiefs obtenus représentaient un revenu d'environ 75000 livres/an pour le Prince de Monaco, dont environ 12000 livres/an ${ }^{7}$ pour le seul comté de Carladez, la châtellenie de Mur-de-Barrez étant la plus importante du comté avec environ 3000 livres/an, soit 1/4 des revenus du comté. Cela montre la richesse de ces espaces agricoles d'alors fondée principalement sur l'élevage, la polyculture mais aussi la présence de péages (au col de Curebourse par exemple). En février 1643, la vicomté de Carlat fut érigée en comté de Carladez ${ }^{8}$ (voir illustration 2), lequel fut complété par l'adjonction des baronnies de Calvinet et la Vinzelle ainsi que par trois châtellenies prises sur la vicomté de Murat ${ }^{9}$. Les adjonctions à la vicomté de Carlat, quand elle fut érigée en comté, complétaient la dotation qui indemnisait Honoré II, nouvel allié de Louis XIII, des conséquences financières de son rattachement à la France.

Illustration 4 - Le texte du traité de Péronne, d'après l'exposition « d'un rocher à l'autre »

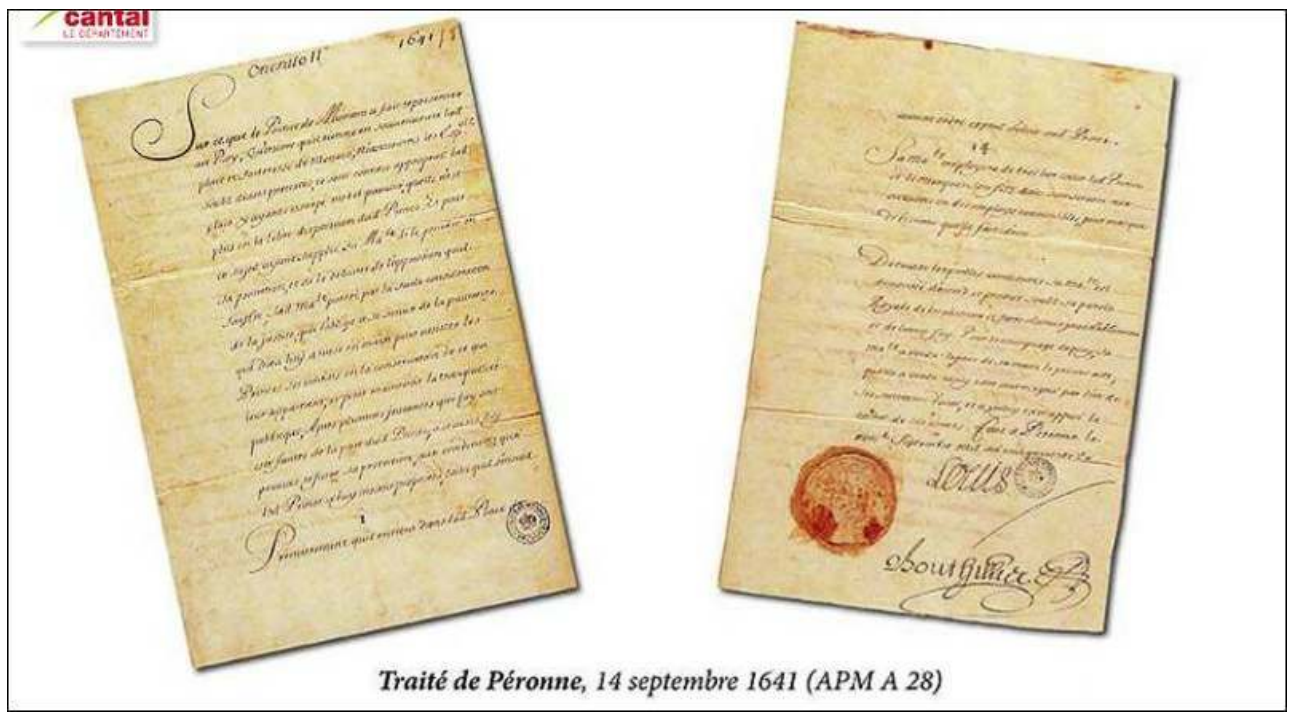

Source : site web des archives départementales du Cantal - http://archives.cantal.fr/?id=684

Du 10 au 25 novembre 1643, Honoré II prenait officiellement possession du comté de Carladez. Pour le représenter lors de la prise de possession, il délégua ses pouvoirs à l'intendant de ses maisons en France. Le commissaire du roi et le représentant du Prince arrivèrent à Vic le 10 novembre 1643 pour y accomplir les formalités administratives qui se renouvelèrent à Mur-de-Barrez puis à Calvinet, chefs-lieux des juridictions principales du nouveau comté. Lors de cette prise de possession, les habitants, par la voix de leurs consuls, demandèrent au nouveau comte la confirmation de leurs droits, franchises, immunités et privilèges qui prévalaient précédemment. Pendant toute la durée de l'administration du comté par les Princes de Monaco, le Carladez connu une certaine prospérité - dans son discours du 14 mai 2014, le maire de Mur-de-Barrez évoquait 
« 148 ans de félicité et de paix, parenthèse sublime, entre deux pages d'histoire noires et sanglantes » - mais qu'il ne faut pas sur-interpréter : elle correspond au «beau XVIII siècle $»^{10}$ que connaît alors l'ensemble du royaume ${ }^{11}$.

9 À l'exception du séjour de Louis I ${ }^{\text {er }}$ à Vic en 1668, où il resta un mois, les princes de Monaco furent des seigneurs absents en Carladez. Ils ne s'y intéressèrent que pour que leur comté rapporte le revenu promis par le roi. L'administration du comté par les intendants du Prince à Paris semblait consciencieuse, les comptes étaient vérifiés scrupuleusement afin d'éviter les abus des fermiers généraux, pratique courante sous l'Ancien Régime; c'est dans ce but que des intendants du Prince viennent sur place contrôler fermiers et sous-fermiers chargés de percevoir les redevances. Les princes de Monaco ne disposaient pas de résidence et ne faisaient donc pas œuvre de charité auprès des habitants comme ce fut le cas dans leurs domaines de Normandie par exemple. Cependant, de 1643 à 1789, on ne signala aucune jacquerie ou doléance particulières des habitants envers leur comte. Les revendications des cahiers de doléances de 1788 sont semblables à celles du reste du royaume, les habitants du Carladez réclament la fin des privilèges, c'est-à-dire l'égalité fiscale, l'égalité civile et la liberté politique.

L'abolition des droits féodaux par l'Assemblée Nationale dans la nuit du 4 août 1789 supprimait presque la totalité des clauses du traité de Péronne et donc les revenus qui en découlaient. Honoré III, Prince de Monaco, était réduit à l'état de simple citoyen. Le Prince fit cependant valoir que ses droits sur le Comté de Carladez ne reposaient pas sur une possession purement seigneuriale, mais qu'ils étaient nés d'un traité diplomatique avec la France et que celle-ci, puisqu'elle ne respectait pas ses engagements, devait lui régler une indemnité. L'assemblée législative reconnut ses prétentions, mais la prise des Tuileries, le 10 août 1792, interrompit les négociations. Les princes de Monaco avaient définitivement perdu leurs fiefs dans le royaume de France.

Or les revenus des domaines du royaume de France permettaient aux princes de Monaco de tenir leur rang à la cour et à Monaco. Leur perte pendant la Révolution française marquait le début d'une période critique pour la Principauté, remettant en cause son existence-même, faute de ressources propres suffisantes. Il faudra attendre le développement $\mathrm{du}$ tourisme balnéaire et des casinos dans la deuxième moitié $\mathrm{du} \mathrm{XIX}^{\mathrm{e}}$ siècle pour que la Principauté retrouve son assise financière. Si la première tentative de création de maison de jeu date de 1856, sous Charles III de Grimaldi, le projet ne fut un succès qu'à partir de 1863 (moment où la présidence de la Société des bains de mer fut attribuée à François Blanc). La perte des territoires de Menton et Roquebrune (1860) conduisit à repenser l'économie monégasque en la dissociant d'un sol devenu plus qu'exigu, la Principauté de Monaco ayant alors vu sa surface réduite de plus de $90 \%$ et s'étant vue privée de ses principaux revenus agricoles. Pourtant, dès les années 1870, la Principauté pouvant suffire à ses besoins, Charles III supprima les impôts personnels, fonciers et mobiliers, ce qui entraîna une intense activité de construction. Dès lors, le rocher méditerranéen attira une clientèle internationale, encore favorisée par le développement de la ligne de chemins de fer Paris-Lyon-Méditerranée arrivée à Monaco en 1868. En 2011-2012, le secteur des jeux a rapporté 173 millions d'euros à la Principauté (IMSEE, 2013, p. 50), le fameux casino de Monte Carlo ne participant que pour moins de $4 \%$ au budget de l'État. Pourtant, il incarne la logique ressource-espace de la mise en exploitation de ressources artificielles (jeux et fiscalité avantageuse), déterritorialisées et extraverties (le casino est interdit aux sujets monégasques) à la suite de la perte des fiefs en 1791 et la rétraction territoriale du XIX'e siècle. 

plus en plus étroits depuis les années 1990. En effet, le maire et conseiller général de Vicsur-Cère prend de nombreuses initiatives pour resserrer les liens entre le Cantal et Monaco. En 1994, il fait réaliser, pour la salle du conseil municipal, un tableau figurant la prise de possession du comté par le représentant du prince Honoré II. En 1997, le Prince Albert II est venu à Aurillac afin de participer à un match de football caritatif; la même année, les maires de Vic-sur-Cère, Carlat, Calvinet et Mur-de-Barrez sont invités pour le 7 e centenaire de la dynastie Grimaldi à Monaco. En 2011, les archives du Palais de Monaco concernant le Carladez sont numérisées et mises en ligne sur le site des archives départementales du Cantal. En 2015 devrait être mis en place un circuit de randonnée cycliste entre le Carladez et Monaco.

Depuis les années 1990, les 150 ans d'administration du Carladez par les princes de Monaco sont mis en valeur par les communes qui comptent en faire, entre autres, un atout touristique.

\section{Du fief au territoire touristique : une destination « Carladez/s »?}

Si la distinction orthographique témoigne d'une forme d'esprit de clocher se traduisant dans l'appartenance départementale, elle manifeste aussi des sentiments ambigus d'appropriation de l'espace: on est du Carladez/Carlades mais aussi fier de marquer sa singularité aveyronnaise ou cantalienne. Comme a voulu l'afficher ce guide touristique en imprimant un tee-shirt pour l'occasion de la visite du Prince Albert II à Carlat, le Carladez/s est une destination qui fait sens. «Il existe une véritable identité du Carladez autour de l'héritage historique et cela attire les touristes qui nous sollicitent pour avoir des informations $»^{12}$ (voir illustration 5). La médiatisation de cette visite a d'ailleurs fait espérer une fréquentation accrue pour l'été 2014, notamment fondée sur la fascination pour les affaires de majesté. Cette information erronée, publiée dans la presse avant la visite, révèle la persistance du fantasme princier : " pour visiter ses anciennes terres et saluer les habitants, selon le programme établi, le Prince Albert II devrait se déplacer à cheval » (La Dépêche du Midi, 12/05/2014) ; belle image d'Epinal de seigneur de retour sur son fief, accompagné de sa princesse... 


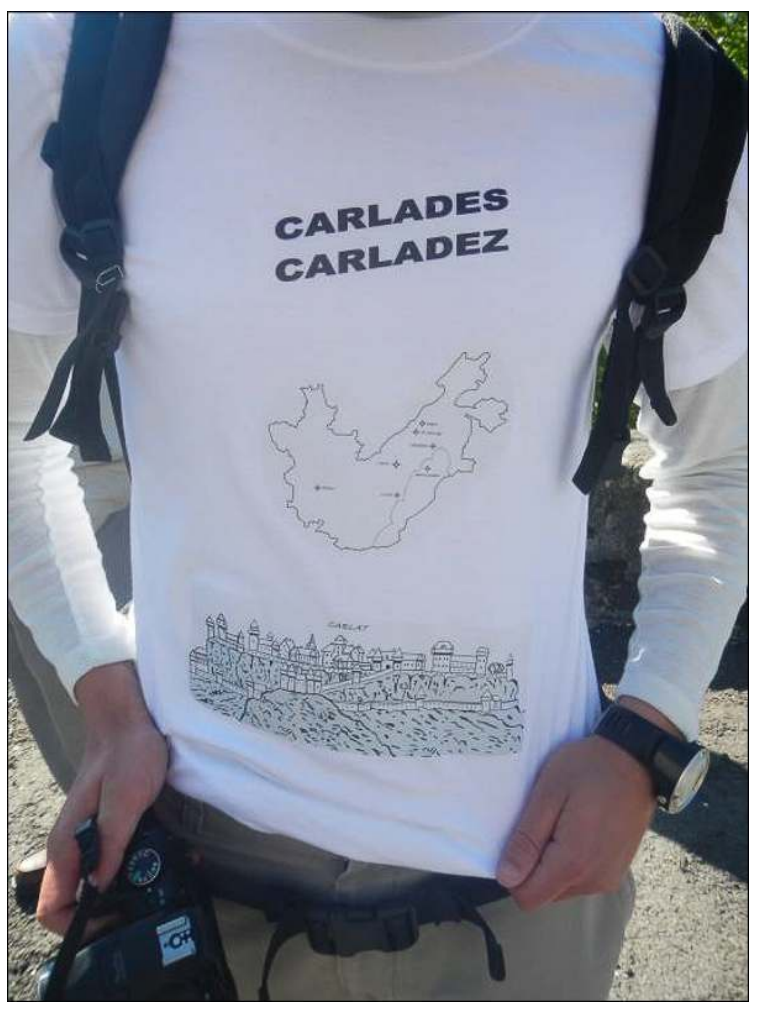

Auteur : M. Redon, 15 mai 2014.

Le Carladez illustre bien que «désignant l'espace perçu comme cohérent par des visiteurs, avec un périmètre plus ou moins défini, pouvant transgresser des limites administratives ou fonctionnelles, relevant à la fois des pratiques et des imaginaires, la destination touristique est en même temps un enjeu des politiques publiques et privées du tourisme, tant en terme de communication que d'aménagements » (Gravari-Barbas, Jacquot, 2012, p. 4). Parmi les territoires touristiques déjà existants, les parcs naturels des volcans d'Auvergne (au Nord) et de l'Aubrac (à l'Est) «prennent en tenaille ", pour reprendre les propos du Maire, le canton de Mur-de-Barrez qui semble avoir du mal à se positionner, d'autant que la tendance actuelle est à la rationalisation, au sein des départements, des syndicats d'initiatives d'intercommunalité. Selon les discussions en cours, le syndicat d'initiative de Mur-de-Barrez se retrouverait en réseau avec ceux des cantons d'Entraygues, d'Estaing, d'Espalion et de Bozouls, un vaste espace le long de la Truyère et du Lot. Autant la mutualisation et la rationalisation des moyens prend tout son sens dans une volonté de gestion rigoureuse des dépenses publiques, autant cet espace manque sensiblement d'identité et ne peut en aucun cas constituer une "destination touristique » alors que le Carladez, administrativement scindé, montre une véritable cohérence des paysages et de l'architecture ainsi qu'une histoire commune. La preuve en est la difficulté à trouver un nom à cette entité touristique disparate, tâche confiée à une société de communication. Sur le terrain, les syndicats d'initiatives de Mur-de-Barrez et de-Vic-sur-Cère ont peu de contacts. Ils orientent respectivement les touristes vers le Carladez ou Carlades mais ne mènent pas d'actions communes, tout en les appelant de leurs vœux: les logiques et politiques départementales prennent le dessus. Ainsi 
l'Aveyron met l'accent sur le « Haut Rouergue » et le Cantal sur son appartenance au PNR des volcans d'Auvergne.

Comme le note le Conseiller général du canton de Mur de Barrez, la Truyère, qui était l'ancienne frontière orientale du Carladez, aujourd'hui « pourrait faire le lien entre les deux départements ». Depuis le printemps 2014, la vidange du barrage de Sarrans sur cette rivière qui fait limite et lien entre les deux départements (voir illustration 2) a fait naître un paysage inédit et temporaire, qui a donné lieu à une fréquentation touristique importante durant l'été 2014, puisque destiné à disparaître de nouveau sous les flots à l'automne. Si «la décentralisation a multiplié les politiques territoriales de développement touristique mais aussi les reconfigurations territoriales » (Gravari-Barbas, Jacquot, 2012, p.4), ces politiques entrent parfois en discordance avec les logiques spatiales qui font localement sens, ignorant les frontières départementales et régionales. Toujours du point de vue du Conseiller général, cette visite a été, incontestablement, "une opportunité en termes de communication, grâce à une forte médiatisation à l'échelle nationale, régionale et dans le département : même à Rodez, ils ne savaient pas ce que le Prince venait faire là, ils ne connaissaient pas cette histoire commune et en étaient très curieux » (entretien du 15/05/14).

La réactivation de l'entité territoriale du Carladez d'Ancien Régime lui confère une visibilité à la fois sur place et à l'extérieur : cela semble relever d'une tendance plus générale à valoriser des identités territoriales niées par les découpages postérieurs.

\section{Le Carladez : un exemple de tensions entre logiques fonctionnelles et découpages territoriaux}

Le Carladez présente une trajectoire particulière en termes de découpage: en 1789, l'ancien fief des Grimaldi s'est trouvé scindé entre deux départements. En 2014, la visite princière a contribué à réactiver les discussions sur la pertinence de l'entité dans un contexte national de remise en cause des découpages territoriaux.

\section{L'instauration d'une limite départementale}

La loi du 22 décembre 1789, instituée le 4 mars 1790, a créé 83 départements; on en compte aujourd'hui 101, outremers compris. D'après Marie-Vic Ozouf Marignier ${ }^{13}$, l'Assemblée constituante a d'abord imaginé des découpages géométriques, le chef-lieu (puis préfecture) étant attribué à une ville située au centre (pour assurer l'aller-retour en une journée à cheval et une à pied pour les sous-préfectures), puis elle a organisé des consultations de l'opinion. Mais la population, encore dans l'esprit des Cahiers de doléances, s'était manifestée auparavant : environ 1500 villes ont envoyé des députés et des dizaines de milliers de villages des représentants pour faire entendre les opinions sur les solidarités territoriales, exprimer la manière dont on dépend d'une ville, dont on va au marché, etc. Dans cette perspective, les difficultés des communications, notamment durant les mauvaises saisons, furent un leitmotiv, en témoigne le cas de Mur-de-Barrez. Aux limites des départements du Cantal et l'Aveyron, cette commune a fait l'objet de discussions : à quel département, et donc à quel chef-lieu la rattacher?

En 1791, une lettre de protestation des députés Lambel et Bô faisait état de la difficulté de se rendre jusqu'à Aurillac et s'opposait donc au rattachement de la commune au Cantal : 
«Je prends le compas, et d'après la carte de Monsieur Cassini, je trouve en planimétrie 4 lieues et demi du Mur-de-Barrez à Aurillac, 21 côtes bien dessinées et 9 rivières ou ruisseaux sans pont. D'après cela, je conclus que dans un pays si raboteux, chaque lieue à vol d'oiseau en vaut et rend au moins 2 pour le voyageur, qu'ainsi, il y a 9 lieues du Murde-Barrez à Aurillac. Maintenant, il faut savoir quelle est la nature de ces côtes et de ces rivières pour les franchir tous les ans au mois de novembre pour les élections et dans toutes les saisons pour suivre les affaires civiles ou d'administration. Ainsi, 21 fois tantôt descendu au sein de la terre et tantôt élevé au-dessus de tout ce qui l'entoure, passant de la sueur à un froid glacial et de l'un à l'autre, le voyageur téméraire arrive après 7 heures des plus pénibles efforts à une lieue d'Aurillac». Implacable démonstration de l'incohérence d'un rattachement du bourg à cette préfecture, et donc au Cantal. Pour reprendre les termes de Roger Brunet, la géographie est bien ici "un inépuisable fournisseur de légitimité » (Brunet, 1997, p. 252) ; les députés Lambel et Bô eurent gain de cause.

Cette limite administrative a ensuite représenté un réel obstacle, comme le montre la savoureuse anecdote des vaches du Père Robert «qui allait, grâce à l'ensemble de la presse catholique, amuser la France toute entière pendant de longs mois " (Beaufrère, 1947, p. 36). Alors que l'institution religieuse des sœurs franciscaines de la Devèze installée à la confluence de la Truyère et du Brezons (voir illustration 2) rencontre des difficultés financières au début du XIXe siècle et que « l'administration veut en finir », les huissiers aidés de bouviers envoyés sur place pour saisir les vaches de l'institution, administrée par le Père Robert, interrogent : «Et les vaches? Toujours affable le Père Robert les leur montre. Elles sont là, plus nombreuses que jamais, à quelques pas, mais de l'autre côté du Brezons, et donc sur le territoire de l'Aveyron où les huissiers ne peuvent instrumenter»(idem, p. 36). La limite administrative a donc bien fait frontière, malicieusement utilisée pour mettre des vaches hors d'atteinte des huissiers. Mais elle représente aujourd'hui une épreuve de gestion quotidienne dans la vie locale.

\section{Un hiatus territorial dénoncé par les élus qui en appellent au «bon sens territorial »}

22 Les problèmes de rattachement liés à la situation de confins ne sont pas nouveaux. Déjà, au moment du Traité de Péronne de 1643, la majeure partie des terres appartenait à l'Auvergne, à l'exception de la châtellenie de Mur-de-Barrez et de la seigneurie de la Vinzelle qui appartenaient au Rouergue (actuel Aveyron), et étaient du ressort du Parlement de Toulouse et non du Parlement de Paris comme le reste des terres concédées. Rappelons que «la France d'Ancien régime était un tissu territorial d'une grande complexité. Les autorités administratives, religieuses, militaires, juridiques imposaient leurs propres limites en une imbrication extrême et difficile à gérer de généralités, diocèses, baillages, eux-mêmes hérités des anciens fiefs (Ardillier-Caraz, 1999, p. 74). Deux siècles après la révolution et sa volonté de rationaliser l'administration, certains enchevêtrements hérités de l'Ancien Régime sont toujours d'actualité : juridiquement, le département de l'Aveyron dépend ainsi de la cour d'appel de Montpellier et non de celle de Toulouse, pourtant capitale de la région. Ces découpages administratifs posent quotidiennement des problèmes de gestion. depuis le canton de Mur-de-Barrez. En ce qui concerne le projet régional de santé, il 
apparait évident aux élus locaux que le canton doit être rattaché au centre d'Aurillac plutôt qu'à Espalion (au sud) et les raisons évoquées sont à l'inverse de celles des députés de 1791 : «au-delà des limites départementales, la ville d'Aurillac se trouve à $40 \mathrm{~km}$ et $30 \mathrm{mn}$ de trajet, alors que la ville d'Espalion est distante de $56 \mathrm{~km}$ pour $55 \mathrm{mn}$ de trajet $»^{14}$. Dans le domaine de l'éducation, les écoles et le collège du canton de Mur-de-Barrez dépendent de l'académie de Toulouse, mais $80 \%$ des élèves de $3^{\mathrm{e}}$ du collège poursuivent leur scolarité dans les lycées d'Aurillac, qui dépendent de l'académie de ClermontFerrand. Indépendamment des directives de leurs rectorats respectifs, les chefs d'établissement des deux départements se concertent afin de faciliter les modalités d'inscription des élèves qui doivent changer d'académie. Une autre difficulté majeure concerne le recrutement des professeurs du collège de Mur-de-Barrez, notamment les remplaçants, qui rechignent à se rendre dans le Nord du département, à plus d'une heure de route de Rodez, alors que des professeurs résidents à Aurillac seraient plus enclins à se déplacer. A la demande « répétée et insistante » du Conseiller général du canton, les deux rectorats semblent ouverts à un assouplissement dans la gestion de leur personnel afin d'autoriser des enseignants remplaçants habitant le Cantal à se rendre à Mur-de-Barrez (entretien avec le Conseiller général canton de Mur-de-Barrez, le 15/05/14). Même cas de figure pour le financement du transport scolaire pour les élèves de la commune de Raulhac située dans le Cantal, qui se rendent au collège de Mur-de-Barrez, ou des élèves de l'école maternelle et primaire de Thérondels, qui habitent les villages voisins situé dans le Cantal (voir illustration 2). Le transport scolaire étant une prérogative des départements, dans la logique administrative, ceux-ci ne doivent pas financer des trajets pour se rendre dans des établissements scolaires d'un autre département. Ici encore, les élus locaux font preuve de pragmatisme et «bricolent» des aménagements exceptionnels.

Parce que le temps de trajet est plus court, les habitants du canton de Mur-de-Barrez ont donc l'habitude de recourir aux services médicaux d'Aurillac, d'y envoyer leurs enfants au lycée, de s'y rendre pour faire leurs achats ; et contrarier ce mouvement semble contraire aux pratiques quotidiennes, à l'espace vécu. La visite du Prince Albert II est venue raviver les discussions sur la pertinence de ces « découpages » administratifs, terme dont Roger Brunet notait la connotation bouchère, donnant l'impression «que le commentateur brandit le tranchoir "; " on découpe de toutes parts, comme s'il s'agissait de s'approprier l'espace, et comme si le territoire n'était que sa carte» (Brunet, 1997, p. 251). Ici, des décennies après les coupures et les découpes, une forme de bon sens territorial semble résister.

La venue d'Albert II est présentée comme "un évènement significatif mais pas déterminant » qui a permis « la prise de conscience de former une entité à présent éclatée mais qui était riche, libre et unifiée. Aujourd'hui, il n'y a pas de rivalités au sein du Carladez mais pas de considération non plus, on trouve cela " amusant » cette différence, ce « $\mathrm{Z}$ » ou ce « $\mathrm{S}$ » à la fin du nom $»^{15}$. Le discours d'accueil du maire a été sans ambiguïté sur le sujet, évoquant les réformes territoriales annoncées et l'émergence de l'entité nouvelle qui se dessine, les "bassins de vie», "espaces de proximité dans lesquels pourraient se retrouver des peuples qui autour d'une histoire commune, partagent les mêmes aspirations et les mêmes projets de vie." Cette notion de bassin de vie a été redéfinie par l'INSEE en 2012 comme le " plus petit territoire sur lequel les habitants ont accès aux équipements et services les plus courants. Son principal intérêt est de décrire les espaces non fortement peuplés, c'est-à-dire les bassins de vie construits sur des unités 
urbaines de moins de 50000 habitants $»^{16}$. Le bassin de vie de Mur-de-Barrez ainsi délimité comprend 9 communes : les 6 du canton, ainsi que celles de Cantoin et SainteGeneviève-sur-Argence en Aveyron et de Raulhac dans le Cantal. Alors le Carladez pourrait, «bien sûr !", reformer une entité cohérente après avoir subi les ciseaux du jacobinisme: "territoire unique et homogène pendant longtemps, le Carladez est aujourd'hui éclaté sur deux départements et sur deux régions différentes. Pris en tenaille entre le parc des volcans d'Auvergne et le Parc de l'Aubrac notre territoire confirme bien qu'il est éternellement " ce petit bout de terre loin de tout ", alors qu'il aurait largement les moyens d'être le centre de lui-même, de devenir le cœur de son propre «bassin de vie » et l'artisan de son propre développement. Serait-ce folie que d'aspirer à redevenir un territoire unifié, à l'image de ce qu'il était à l'époque de votre gouvernance ? Cette réunification du Carladez est-elle souhaitable ? Réalisable?»

Pour l'heure, les communes du canton sont regroupées dans la Communauté de communes du Carladez tandis que celle du canton de Vic-sur-Cère forment celle des communes de Cère et Goul en Carladès, à l'exception de la commune de Carlat, qui donne pourtant son nom à l'ensemble mais a préféré adhérer à la Communauté d'agglomération du Bassin d'Aurillac étant donné que la majorité de ses actifs y travaillent (voir illustration 2). Le projet de loi sur les découpages territoriaux contraindrait aux rassemblements de communautés de communes (EPCI) devant comporter 20000 habitants, au lieu de seulement 5000 aujourd'hui. Or on compte à présent un total de moins de 8500 habitants pour le Carladez : 5388 habitants pour le canton de Vic-sur-Cère et 2950 du côté du canton de Mur-de-Barrez ${ }^{17}$. Un grand Carladez réunifié pourrait alors être à l'ordre du jour, les limites départementales n'étant pas un obstacle aux EPCI et les élus ne semblant pas y être opposés ${ }^{18}$. A devoir créer des entités plus vastes et peuplées, l'ancien fief fait sens et identité, même si l'agglomération d'Aurillac polarise indéniablement l'espace.

\section{Conclusion}

" On trouvera à tout raboutage sa légitimité; mieux, une « vocation » redécouverte ; une bénédiction. Ce qui compte est pourtant la raison du regroupement, plus même que la rationalité des sutures. En général, on s'assemble pour être fort» (Brunet, 1997, p. 254). La question du regroupement se pose avec d'autant plus d'acuité dans les espaces ruraux reculés du Carladez. "Pour les intercos aussi, "big is beautiful" » dénoncent les maires sur le site du Courrier des maires et des élus ${ }^{19}$. Si la tendance est à la formation d'intercommunalités plus grosses, afin d'obtenir des masses critiques de population dans une France potentiellement à 14 régions, jusqu'où peut s'étendre la pertinence des regroupements spatiaux et sur quelle base les fonder: la logique du nombre, celle des espaces de vie quotidienne, la nécessité d'exister aux yeux de l'extérieur? Les légitimités territoriales varient dans le temps et l'espace mais il est indéniable que certains découpages pré-jacobins font sens et territoire. Même en cas d'adoption du plan de redécoupage des régions, le Carladez sera toujours scindé entre deux régions; or la référence commune aux Grimaldi de Monaco constitue un ferment d'unité que la visite du mois de mai 2014 a fortement réactivée. Les enjeux symboliques et mémoriels y sont d'autant plus forts qu'ils distinguent un territoire du Massif central et l'érige en destination, auréolé de l'imaginaire princier. 


\section{BIBLIOGRAPHIE}

Ardillier-Carras F., 1999. Les pays et l'espace vécu. Quelles logiques pour quels territoires? Norois, $n^{\circ} 181,1999-1$, « Pays » et développement local. Logique et ambiguïté d'une politique des territoires, p. 173-181.

Banque de France, 2014. Zone euro. Principaux indicateurs économiques et financiers, Fascicule consultable en ligne, 40 p. https://www.banque-france.fr/economie-et-statistiques/base-dedonnees/chiffres-cles-de-la-zone-euro.html

Beaufrère A., 1947. Les sœurs franciscaines de la Devèze. Editions Gerbert, Aurillac, 110 p.

Boudartchouk J.-L., 1998. Le Carladez de l'Antiquité au XIII siècle. Territoire, homme et pouvoirs. Thèse, Université de Clermont.

Brunet R., 1997. Territoires : l'art de la découpe/Pinking shears applied to territories. Revue de géographie de Lyon, Vol. 72, $\mathrm{n}^{\circ}$ 3, Les ciseaux du géographe : coutures et coupures du territoire, p. 251-255.

Documents relatifs à la vicomté de Carlat, recueillis et publiés par ordre de S.A.S. le Prince Albert Ier par Gustave Saige et le Comte de Dienne, 1900, Monaco ; réédition Aurillac, 2007, 2 in-8.

Farges L., 1927. Le Carladez et le Barrez dans la nature et dans l'histoire. Conférence, Aurillac, USHA, $15 \mathrm{p}$.

Fouilheron J., 2011. Un fief pour une alliance. Les princes de Monaco, comtes de Carladès (XVII ${ }^{\mathrm{e}}$ $\mathrm{XVIII}^{\mathrm{e}}$ siècle). Revue de la Haute Auvergne, dossier « Le rocher de Carlat, entre Auvergne et Monaco : enjeu diplomatique, savant, et mémoriel », t. 73, p. 312-330.

Gravari-Barbas M., Jacquot S., 2012. Les géographes et les métiers du tourisme. EchoGéo, [En ligne], numéro 19 | 2012, mis en ligne le 10 février 2012, consulté le 15 février 2012. URL : http:// echogeo.revues.org/13009

EchoGéo, Sur le Vif 
IMSEE (Institut Monégasque de la statistique et des Études Économiques), 2013. Monaco en chiffres, $55 \mathrm{p}$.

Ozouf Marignier M.-V., 1993. La formation des départements. Éditions de l’EHESS.

Pelletier P., 2014. De nouvelles régions, ou le degré zéro de la géographie. La lettre d'Orion, Libération - vendredi 11 avril 2014 - consultable sur : http://

libelalettredorion.blogs.liberation.fr/mon-blog/2014/04/combien-de-r\%C3\%A9gions-pour-lafrance-.html

Piolet V., 2013. Oui, la Principauté est bien un paradis fiscal. Le Monde.fr du 07/05/2013, consultable sur http://www.lemonde.fr/idees/article/2013/05/07/oui-la-principaute-demonaco-est-bien-un-paradis-fiscal_3173026_3232.html

\section{NOTES}

1. Selon que l'on se trouve dans l'Aveyron ou le Cantal les terminologies diffèrent: " $\mathrm{s}$ " final dans le Cantal et « $\mathrm{z}$ » dans l'Aveyron.

2. Au XVIII ${ }^{\mathrm{e}}$ siècle, les princes de Monaco ont acquis d'autres fiefs en territoire français, en Normandie, dans le Nord-Est, en Ile-de-France et dans le Poitou. Les seigneuries qui provenaient de la succession du prince Jacques I $^{\text {er }}$ de Monaco, fils de Jacques III de Matignon (une des plus anciennes familles de Normandie) qui a épousé en 1715 la princesse Louise-Hippolyte, fille du prince Antoine $\mathrm{I}^{\mathrm{er}}$. À la mort de Jacques III en 1725, il reçut pour la maison de Monaco, la seigneurie de Matignon, le comté de Torigni, les baronnies de Saint Lô, de la Luthumière, Hambye, Moyon, Plessis-Grimoult, la Roche-Tesson. Des fiefs que possédaient les Grimaldi en France, les plus nombreux provenaient du mariage d'Honoré IV avec Louise-Félicité-Victoire d'Aumont en 1777, héritière des biens légués par le Cardinal de Mazarin à Armand Charles de la Porte, époux de sa nièce Hortense Mancini. Ces biens comprenaient le marquisat de Guiscard, le duché de Mayenne, le duché de Rethel-Mazarin, le marquisat de Chilly, le comté de Longjumeau, la baronnie de Massy, les comtés de Ferrette, Belfort, de Rosemont, de Thann, la baronnie d'Altkirch, la seigneurie d'Issenheim, le duché d'Aumont, le duché de la Meilleraye.

3. Entretien avec le directeur des archives et de la bibliothèque du Palais de Monaco (19/05/2014).

4. Le rocher de Carlat a été vendu comme bien national en 1796, après avoir appartenu à différents propriétaires privés. La société savante de la Haute Auvergne en fait l'acquisition en 1911 par crainte d'une destruction du site historique et archéologique avant de le rétrocéder au prince Albert Ier de Monaco en 1914.

5. «L'omniprésence de plus en plus pesante de l'Espagne à Monaco finit par transformer la petite seigneurie, à juste titre soucieuse de son indépendance, en base opérationnelle habsbourgeoise et par menacer la souveraineté de son seigneur et prince » (Fouilheron, 2011).

6. « Les sirènes française, orchestrées par le Cardinal de Richelieu, commencent à trouver auprès $\mathrm{du}$ prince et sur le rocher, des oreilles réceptives » (idem).

7. Il est délicat de donner une valeur actuelle à ces sommes. Si les historiens, pendant longtemps, se sont accordés à donner, en corrélation avec la cote de l'or ou celle du blé, une valeur moyenne de $8 € / 2006$ à la livre tournois, l'historien Jean Fourastié proposait de contourner la difficulté due aux variations de la monnaie en employant une unité valable en tout temps et en tout lieu: le salaire horaire, bien difficile à donner ici dans le cas du Comté de Carladez... Mais si l'on se réfère à Emile Levasseur, dans son étude sur « les prix, aperçu de l'histoire économique de la valeur et du revenu de la terre en France du commencement du XIIIe siècle à la fin du XVIIIe » parue en 
1893, une paire de bœufs de travail valait environ 90 livres, une vache et son veau 40, un cochon 25 à la fin du XVIIIe siècle.

8. Lettres patentes portant donation à Honoré II, prince de Monaco, de la vicomté de Carlat et terres adjacentes érigées en comté de Carladez, février 1643 Saint-Germain-de-Laye, Archives du Palais de Monaco, G 1, dossier $n^{\circ} 1$.

9. Vigouroux, Turlande et l'ancien mandement de Barrez composé des paroisses de La Capelle Barres, Malbo et Narnhac.

10. Terme utilisé par les historiens pour décrire la croissance démographique et les progrès économiques constatés en France au XVIII ${ }^{\mathrm{e}}$ siècle ; la notion est reprise et développée dans deux ouvrages de référence: L'Histoire économique et sociale de la France, dirigé par F. Braudel et E. Labrousse (1970) et Histoire de la France rurale, dirigé par G. Duby et A. Wallon (1975).

11. Le climat est moins froid qu'au XVII ${ }^{\mathrm{e}}$ siècle, ce qui permet de meilleures récoltes. Les guerres épargnent le territoire français, le commerce connaît une forte expansion et les grandes crises démographiques sont en net recul (famines moins fréquentes et moins profondes, recul des grandes épidémies et disparition de la peste à partir de 1720). De 1715 à 1789, la population française passe de 22 à 28 millions d'habitants, ce qui fait de la France le pays le plus peuplé d'Europe à la fin du XVIII siècle.

12. Entretien au Syndicat d'initiative de Mur-de-Barrez (14/05/2014).

13. Emission « La marche de l'histoire » du 12/05/2014 consacrée à l'Histoire des départements voir aussi Ozouf Marignier (1993).

14. Extrait de la lettre de Daniel Tarisse, Conseiller général de Mur-de-Barrez adressée le 5 mai 2011 à la Délégation territoriale de l'Agence de santé de l'Aveyron.

15. Entretien avec le maire de Mur-de-Barrez (15/05/2014).

16. D'après l'INSEE, «On délimite ses contours en plusieurs étapes. On définit tout d'abord un pôle de services comme une commune ou unité urbaine disposant d'au moins 16 des 31 équipements intermédiaires. Les zones d'influence de chaque pôle de services sont ensuite délimitées en regroupant les communes les plus proches, la proximité se mesurant en temps de trajet, par la route à heure creuse. Ainsi, pour chaque commune et pour chaque équipement non présent sur la commune, on détermine la commune la plus proche proposant cet équipement. Les équipements intermédiaires mais aussi les équipements de proximité sont pris en compte.» Consultable sur: http://www.insee.fr/fr/methodes/default.asp?page=zonages/bassinvie-2012.htm

17. Alors que la population était estimée à près de 11700 habitants en 1962, dont 4759 habitants pour le canton de Mur-de-Barrez et 6930 pour celui de Vic-sur-Cère (INSEE).

18. Entretiens à la Mairie de Vic-sur-Cère (26/09/2014)

19. http://www.courrierdesmaires.fr/36411/reforme-territoriale-une-nouvelle-mouture-pourmuscler-linterco-et-devitaliser-le-departement/ - projet de loi portant nouvelle organisation territoriale de la République - NOR : RDFX1412429L/Rose-1

20. Idem.

\section{RÉSUMÉS}

L'article a pour point de départ la visite, les 14 et 15 mai2014, du prince Albert II de Monaco dans le Carladez, territoire correspondant au fief échu en 1643 à la famille Grimaldi et à présent scindé 
entre les départements du Cantal et de l'Aveyron, et donc les régions Auvergne et Midi-Pyrénées. Par une approche géo-historique, le propos est de comprendre ce que représente ce lien historique entre ces deux territoires en 2014, à la fois pour la Principauté et les habitants des communes concernées : l'ancien fief fait-il toujours sens, comme entité territoriale, malgré les découpages postérieurs? Qu'est-ce qui donne sa légitimité à un découpage territorial dans un contexte de remise en cause des partages de la République (projet de réforme de la carte des régions en juin 2014) ? Après un tableau comparatif montrant la force des différences entre ces deux espaces, une analyse de la construction des rapports entre ces deux territoires au fil d'une histoire commune est proposée. La mise en place des limites administratives après la Révolution française mène à questionner la relativité de la notion de distance dans le temps et la persistance du fief en tant que destination touristique. L'article tend à montrer que les enjeux symboliques et mémoriels sont d'autant plus forts dans le Carladez qu'ils distinguent un territoire du Massif central et l'érige en destination, auréolé de l'imaginaire princier. Quant à la Principauté de Monaco, elle est emblématique de la logique spatiale selon laquelle l'exception rapporte davantage que la continuité territoriale.

The starting point of this article is Prince Albert II of Monaco's visit to the Carladez district on 14th and 15th May 2014, a district corresponding to the fief fallen to the Grimaldi family's share in 1643 and now straddling Cantal and Aveyron departments and thus the Auvergne and MidiPyrénées regions. Through a geo-historical approach, the intention is to understand the meaning of the historic link between the two territories, Monaco and the Carladez, both for the Principality and for the inhabitants of the communes concerned: does the former fief still make sense as a territorial entity despite its having been split up? What makes legitimate a territorial carving when the Republic's internal borders are being questioned (proposal of a territorial reform to redraw the map of regions in June 2014)? A comparison chart showing how marked the differences are between the two areas is followed by an analysis of the construction of the relations between the two territories in the course of their common history. The setting up of the administrative boundaries after the French Revolution leads to question the relativity of the idea of distance over time as well as the continued existence of the fief as a tourist destination. The article aims to show that the symbolic and memorial issues are all the greater in the Carladez because they set apart a territory of the Massif Central and establish it as a destination with an aura of princely prestige. As for the Principality of Monaco, it is emblematic of the spatial logic according to which the exception is worth more than territorial continuity.

\section{INDEX}

Keywords : territorial entity, territorial carving, administrative boundaries, department ; region, living area, inter-municipal level, tourist destination, viscountcy, county, seigniory, fief, feudalism, vassal, Grimaldi, Albert II, Monaco, Carladez, Aveyron, Cantal.

Mots-clés : entité territoriale, découpages territoriaux, limite administrative, département, région, bassin de vie, intercommunalité, destination touristique, vicomté, comté, seigneurie, fief, féodalité, vassal, Grimaldi, Albert II, Monaco, Carladez, Aveyron, Cantal

\section{AUTEURS}

\section{GÉRAUD CULLIER DE LABADIE}

Géraud Cullier de Labadie, geraudcdl@wanadoo.fr, est Professeur d'histoire et géographie dans l'académie de Toulouse 


\section{MARIE REDON}

Marie Redon, mredon@yahoo.com, est Maitre de conférences en géographie à l'Université Paris 13 et membre de l'EA 2356 CRESC/Pléiade et de l'UMR 8586 Prodig. 